\title{
Erratum to: Identification and Characterization of Four Missense Mutations in Brown midrib12 (Bmr12), the Caffeic acid $O$-Methyltranferase (COMT) of Sorghum
}

\author{
Scott E. Sattler • Nathan A. Palmer • Ana Saballos • \\ Ann M. Greene - Zhanguo Xin • Gautam Sarath • \\ Wilfred Vermerris $\cdot$ Jeffrey F. Pedersen
}

Published online: 10 July 2012

(C) Springer Science+Business Media, LLC 2012

\section{Erratum to: Bioenerg. Res.}

DOI 10.1007/s12155-012-9197-z

The original version of this article unfortunately contained some mistakes. The name of the enzyme "Caffeic $O$-methyltransferase" should be read as "Caffeic acid $O$-Methyltranferase" throughout the paper, including the title.

On Table 2, the fifth column should have been captured as part of the sub-heading "Unpretreated". The corrected version is shown on the next page.

The online version of the original article can be found at http://dx.doi.org/ 10.1007/s12155-012-9197-z.

S. E. Sattler $(\bowtie) \cdot$ N. A. Palmer $\cdot$ G. Sarath $\cdot$ J. F. Pedersen Grain Forage and Bioenergy Research Unit, USDA-ARS,

Lincoln, NE 68583, USA

e-mail: Scott.Sattler@ars.usda.gov

S. E. Sattler · N. A. Palmer · G. Sarath · J. F. Pedersen

Department of Agronomy and Horticulture,

University of Nebraska-Lincoln,

Lincoln, NE 68583, USA

A. Saballos · A. M. Greene $\cdot$ W. Vermerris

Agronomy Department and Genetics Institute,

University of Florida,

Gainesville, FL 32610, USA

\section{Z. Xin}

Plant Stress and Germplasm Development Unit, USDA-ARS,

Lubbock, TX 79415, USA 
Table 2 Variation in Klason lignin content and glucose yields obtained after enzymatic saccharification at $50{ }^{\circ} \mathrm{C}$ at $60 \mathrm{FPU} / \mathrm{g}$ cellulase of native (unpretreated) stover after 4, 20 , and $96 \mathrm{~h}$, and of pretreated stover after $24 \mathrm{~h}$

${ }^{a}$ Means in rows with different letters are statistically significant based on Tukey's HSD with an experiment-wise error rate of $0.05(n=9)$

\begin{tabular}{|c|c|c|c|c|c|}
\hline & \multirow{3}{*}{$\begin{array}{l}\text { Klason lignin }(\mathrm{mg} / \mathrm{g}) \\
\text { Mean (SD) }\end{array}$} & \multicolumn{4}{|c|}{ Glucose yield (mg/g stover) } \\
\hline & & \multicolumn{3}{|l|}{ Unpretreated } & \multirow{2}{*}{$\begin{array}{l}\text { Pretreated } \\
24 \mathrm{~h} \\
\text { Mean (SD) }\end{array}$} \\
\hline & & $\begin{array}{l}4 \mathrm{~h} \\
\text { Mean (SD) }\end{array}$ & $\begin{array}{l}20 \mathrm{~h} \\
\text { Mean (SD) }\end{array}$ & $\begin{array}{l}96 \mathrm{~h} \\
\text { Mean (SD) }\end{array}$ & \\
\hline WT BTх623 & $21.2(1.9) \mathrm{a}$ & $38(6) \mathrm{bc}$ & $54(5) \mathrm{d}$ & $55(4) d$ & $174(25) \mathrm{c}$ \\
\hline bmr12-ref & $17.1(0.8) \mathrm{cd}$ & $42(6) \mathrm{abc}$ & $62(5) \mathrm{cd}$ & $64(8) \mathrm{bcd}$ & $212(19) b$ \\
\hline bmr12-30 & $18.4(0.9) b c$ & 43 (13)abc & $64(7) \mathrm{bc}$ & $70(8) \mathrm{abc}$ & $230(27) a b$ \\
\hline bmr12-34 & $19.3(1.5) \mathrm{b}$ & $51(10) \mathrm{ab}$ & $74(9) \mathrm{ab}$ & $74(9) \mathrm{ab}$ & $213(22) b$ \\
\hline bmr12-820 & $16.7(1.7) \mathrm{d}$ & $54(14) \mathrm{a}$ & $79(12) \mathrm{a}$ & $82(13) a$ & $238(35) \mathrm{a}$ \\
\hline bmr12-35 & $19.6(0.5) \mathrm{ab}$ & $32(7) c$ & $54(6) \mathrm{d}$ & $59(12) \mathrm{cd}$ & $178(12) \mathrm{c}$ \\
\hline HSD (0.05) & 2.2 & 10 & 8.3 & 9.9 & 22 \\
\hline
\end{tabular}

